\title{
Index des personnes
}

Adam, Antoine 135, 140

Adelphes, les, voir aussi Dupuy

111

Albergati 190-191

Alciat, André (Alciato, Andrea) 87

Alexandre 49

Althusius, Johannes 78

Alvares, Nuno 37

Ammirato, Scipione 8, 200-202

Amurat I (Murat I ${ }^{\text {er }} 47$

Anne d'Autriche 109, 121, 147

Apollon 187, 190, 196

Aristote 14, 81, 84, 89, 147, 160, 181

Auguste 47

Automne, Bernard 86

Ayrault, Pierre 19

Azon de Bologne 15

Bagno, Giovan-Francesco Guidi di (De

Bagny, De Bagni), cardinal 154-159, 163-166, 174-175, 177

Balde 25

Baldini, Enzo 37

Barberini, Francesco 170

Bardin, Erard 144

Barillon, Jean-Jacques de 149

Bartole 16

Basile 58

Battista, Anna-Maria 100

Bayezit II 51

Bernard, abbé de Clairvaux 155

Berns, Thomas 81

Bèze, Théodore de $89-90$

Bibliander, Theodor 38,62

Billaut, Adam 143-144

Blickle, Peter 78

Boccalini, Trajano 187, 194

Bodin, Jean (bodinien, bodinienne) 18 , 20-21, 24-26, 34, 42-48, 54-55, 60-62, 77, 80-85, 87, 92, 99, 102, 104-106, 117, 180-192

Botero, Giovanni 56-57, 59, 119, 180, 185-187, 193-197

Boulliau, Ismaël 132

Braudel, Fernand 39

Brisson, Barnabé 7,63

Buckley, Samuel 95

Budé, Guillaume 63, 81

Brutus, Stephanus Junius 89, 94
Cajetan (Gaetani, Constantino) 154-162, $167-169,171,173$

Calvin, Jean 50, 87

Campanella, Tomaso 38

Cervantès 61

César 31, 49

Chalcondyle 54

Chapelain, Jean 135, 145

Chappuys, Gabriel 52-55, 59, 63

Charles-Quint 51, 83

Chasseneuz, Barthélemy de 16-18

Choppin, René 87

Ciceron 81, 110

Cinq-Mars 100, 114, 127, 149

Clément VIII 188, 191-192

Colbert, Jean-Baptiste 149

Colletet, Guillaume 135, 169-170

Colonna, Marc'Antonio 41

Commode 58, 121, 125

Conring, Hermann 106

Contarini, Gasparo 81-82

Coquille, Guy 93, 144

Cosme de Médicis 84

Crosilles, Jean-Baptiste de 135

Della Casa, Giovanni 37

Demante, Georges 108

Denis l'Aréopagite 163

Denys d'Halicarnasse 81, 181

Denis de Paris (saint) 163

Diane 181

Dion 43, 181

Domat, Jean 86

Duchesne, André 145

Du Moulin, Charles 86, 96, 100

Dupuy, Christophe 94

Dupuy, Claude 64

Dupuy, Jacques 152, 157-160, 162-164

Dupuy, Pierre 96, 99-100, 108-109, 112 131

Dupuy, les ou les frères, voir aussi Adelphes 7, 94-95, 98, 106, 108, 111-115, 118-119, 121-122, 130-132, 135, 149, $154,167,174$

Duret, Jean 86

Du Ryer, Pierre 79, 103-104

Eleusis 181

Emilio Paolo 29 
Erasme 99, 112

Estienne, Henri 41

Ferrault, Jean 16

Foglietta, Uberto 40-42

Fonseca, Jeronimo Osório da 37

Fortin de la Hoguette 132

Frachetta, Girolamo 57-59, 200

François $1^{\text {er }} 18$

Fronteau, Jean 158, 167, 170, 174

Fumaroli, Marc 109-110

Furetière, Antoine 148

Gaetani, Constantino, voir Cajetan

Gassendi, Pierre 135

Gaston, duc d'Orléans 113, 115, 123, 127, 143

Georges de Trébizonde 81-82

Georgieviz, Bartholomaeus, 38, 54

Gersen, Jean 152, 154-155, 157, 160-162, 167-168, 171-172, 174-176

Gerson, Jean 155-156, 158, 160, 162-163, $168,172,176$

Gesvres, marquis de 147

Giannotti, Donato 82

Giovio, Paolo, voir Jove, Paul

Godefroy, famille ou les 98, 106

Godefroy, Théodore 99, 114

Gomberville, Marin Le Roy de 138, 144

Gonzague, les ou maison de ou famille de, voir aussi Nevers 134, 138-139, 142, 144

Gonzague-Mantoue, maison de 140

Gonzague, Anne de 140, 142-143

Gonzague, Charles I ${ }^{\text {er }}$ de 142

Gonzague, Marie de, voir aussi Marie, la Princesse 134, 140, 142-143, 145

Goujet, Claude-Pierre 136, 139

Gournay, Marie de 135

Grassaille, Charles de 16-17

Grégoire, Pierre dit Toulousain 34-35

Grégoire XIII 162, 168

Henri de Navarre 57

Henri III 53

Henri IV 191

Henri VI (empereur) 15

Hotman, François 23, 28, 30-35, 87-92, 96-97, 101

Huet, Pierre-Daniel 135

Jove, Paul (Giovio, Paolo) 38, 59, 62, 119

Juan d'Autriche 51
Kantakouzène 38

Koenigsberger, Helmut 22

Lampadius, Jacob 106

Languet, Hubert 76-79, 87, 101, 106

La Forest, Jean de 39

La Mothe Le Vayer, François 112, 132, $135,169-170$

Launoy, Jean de 147, 163, 172

Laurière, Eusèbe 86

La Vigne, Jean de 43, 48

Le Bret, Cardin 117

Léon l'Africain 37

Le Roy, Louis 32-35

L'Estoile, Pierre de 35

Le Voye (Le Voix), Loyse 64

L'Hommeau, Pierre de 86

Lipse, Juste 61

Lodewijcksz, Willem 37

Lothaire de Crémone 15

Louis XI 90, 125

Louis XII 58

Louis XIII 109, 113, 118-119, 124, 126$128-129,148$

Louis XIV 60, 144, 147

Louvet, Pierre 86

Loyseau, Charles 16

Lucinge, René de 49-51, 56

Luther 38, 50, 62

Lycurgue 82

Machiavel 37, 180-182, 184-190, 192, 194, 196, 202-203

Machault, Jean-Baptiste 74

Machault, Jean 96

Mantoue et de Nevers, maison de, voir aussi Nevers 139

Marillac, Charles de 22, 34

Marolles, Michel de 133-150

Marie de Médicis 125

Marie, la Princesse, voir aussi Gonzague, Marie de 134, 140-145, 149

Martinellii, Fioravante 156, 174

Masparraulte, Pierre de 64

Mattei, Domenico 37

Maucors, Piat 135

Maximilien 83

Mayenne, duc de 57

Mazarin 118, 121, 130, 132, 144, 148-

149, 167

Mehmet II 38, 46-47, 49, 60, 62,

Mehemet (Mahemet) 44, 46

Meinecke 37 
Melanchthon 38, 62

Melliet, Laurens 200-201

Michelet 108

Molé, Mathieu 112

Molière d'Essertines, François-Hugues Forget de 135

Montaigne, Michel de 33, 59, 99

Montholon, François de 141

More, Thomas 52, 54, 112, 186

Moreau-Reibel, Jean 81

Naudé, Gabriel 7, 132, 152-174, 177-178, 197-199, 202-203

Nevers, ducs de ou maison de, voir aussi Gonzague; Mantoue et de Nevers 134, $138,141-142$

Nicolay, Nicolas de $37-38,59$

Orcibal, Jean 108

Panormitain 18

Pasquier, Étienne 23-25, 31, 118

Pastoureau, François 65

Patin, Guy 132, 135, 155, 161, 163

Pétrarque 110, 112, 119

Piccolomini, Enea Silvio 28

Pie II 28, 38, 62

Platon, platonicien, platonicienne 14,20 21, 33, 81-82, 89, 186

Plutarque 181, 202

Poille, Jacques 73

Polybe 181

Postel, Guillaume 30, 39-40, 49, 54, 59, 63-64, 81

Prévost d'Exiles, Antoine-François 79, 104-105

Prévost, Bernard 63

Ptolémée le Flûtiste 48

Quatremaires, Robert 168-169

Quintilien 19

Racine, Jean 61

Ramusio, Giovanni-Battista 37

Ramée, Pierre de la 30-31

Reffuge, Eustache du 73

Régnier de la Planche, Louis 22

Retz 109, 131

Ribadeneyra 184-185

Richelieu 9, 99-100, 108-109, 113-116, 118-119, 121-132, 143, 148-149, 154$160,163,165$
Rigault, Nicoles 112, 132

Rivière, Denis de 63

Ronsard, Pierre de 30, 33

Rousseau, Jean-Jacques 78

Rustem Pacha 48

Saint André, Pierre de 63

Sansovino, Francesco 51-55,

63

Séguier, Pierre 146, 149, 159

Selim 49, 51

Sénèque 110

Seyssel, Claude de 88-89, 118

Sinan Pacha 44, 59

Sirmond, Jacques 163

Sixte Quint 191

Skalweit, Stefan 77-78

Sorbière, Samuel de 135, 149

Spandounes, Théodore 51-54

Strada, Famien 152

Suleyman 44, 48-49, 52

Suriano, Michele 21

Tacite $61,129,181,184-185,189,194$, 201-202

Tallemant des Réaux, Gédéon 135

Théodose le Grand 46-47

Thomas a Kempis 154-158, 162, 167$168,170-172,174$

Thou, Christophe de 91, 99

Thou, François-Auguste de 100, 114, 121122,127

Thou, Jacques-Auguste de 61-63, 65, 76, 79, 91, 93-106, 111, 113

Thou, les de 7,10,132

Tite-Live 81

Toledo, Francisco 191

Tschudi, Aegidius 29

Thucydide 81

Ventura, Comino 55

Viala, Alain 140

Viole, Jacques 65

Viroli, Maurizio 37

Wolsey 120-121, 125

Xiphiline 43

Zarka, Yves-Charles 37

Zuber, Roger 99, 140 